\title{
THE METHOD OF FACTORIZING THE FUNCTION OF THE DISTRIBUTION OF BIDIRECTIONAL REFLECTION IN THREE-DIMENSIONAL SCENES
}

\author{
МЕТОД ФАКТОРИЗАЦИИ ФУНКЦИИ РАСПРЕДЕЛЕНИЯ \\ ДВУНАПРАВЛЕННОГО ОТРАЖЕНИЯ В ТРЁХМЕРНЫХ СЦЕНАХ
}

\section{МЕТОД ФАКТОРИЗАЦІЇ ФУНКЦІЇ РОЗПОДІЛУ ДВОНАПРАВЛЕНОГО ВІДОБРАЖЕННЯ В ТРИВИМІРНИХ СЦЕНАХ}

\author{
Olexandr N. Romanyuk \\ rom8591@gmail.com \\ ORCID: 0000-0002-2245-3364 \\ Sergey I. Vyatkin \\ sivser@mail.ru \\ ORCID: 0000-0002-9346-145X \\ Viktoriia V. Voitko \\ dekanfki@i.ua \\ ORCID: 0000-0002-3329-7256 \\ Oksana V. Romanyuk \\ romaniukoksanav@gmail.com \\ ORCID: 0000-0003-0235-8615
}

А. Н. Романюк ${ }^{1}$,

докт. техн. наук, профессор

С. И. Вяткин ${ }^{2}$,

канд. техн. наук, с. н. с.

В. В. Войтко ${ }^{1}$, канд. техн. наук, доцент

О. В. Романюк ${ }^{1}$, канд. техн. наук, с. н. с.

\begin{abstract}
${ }^{1}$ Vinnytsyia National Technical University, Vinnytsia
${ }^{1}$ Винницкий национальный технический университет, г. Винница

${ }^{1}$ Вінницький національний технічний університет, м. Вінниця

${ }^{2}$ Institute of Automation and Electrometric of the Siberian Branch of the Russian Academy of Sciences, Novosibirsk

${ }^{2}$ Институт автоматики и электрометрии Сибирского отделения Российской академии наук, г. Новосибирск ${ }^{2}$ Інститут автоматики і електрометрї Сибірського відділення Російської академії наук, м. Новосибірськ
\end{abstract}

\begin{abstract}
The bidirectional reflection distribution function describes the reflection of light from a surface. It is important to use arbitrary bidirectional reflections when rendering in real-time. The aim of the work is to increase the realism of smooth surfaces using the factorization of the distribution function of bidirectional reflection in three-dimensional scenes, which allows us to adapt the process for use by modern graphic hardware processors. The bidirectional reflection representation compression method should be applied using available texture mapping and GPU computing capabilities. The scientific novelty of the work lies in the proposed numerical method of homomorphic factorization, with which it is possible to decompose arbitrary bidirectional reflections into products of two or more factors of smaller dimensions, each of which depends on the interpolated geometric parameters. Compared to the well-known factorization method based on the decomposition of singular values, the proposed method generates factorization only with positive factors. This difference is of great practical importance because it makes it more suitable for modern graphic hardware processors, providing control over the smoothness of the resulting surface, minimizing relative rather than absolute error, and can deal with scattered, sparse data without a separate sampling and interpolation algorithm. The physical reflection coefficient of a surface can be modeled locally using the bidirectional reflectance distribution function method. For a uniform surface, the outgoing radiation from a point in a certain direction can be calculated using the integral of the incoming radiation over all incoming directions. A numerical approach based on a singular expansion constructs an approximation of a series consisting of the sum of several products of functions. In contrast to the numerical approach, the proposed method uses a pure product of functions during factorization. The projections of the functions determine the parameterization of the factors with respect to the original parameterization of the bidirectional reflection. Projections of functions can also be non-linear. The proposed shared representation of the function avoids negative numbers, which allows the use of simple and easily computed parameterizations. This ensures the flexibility of the proposed method and determines the ease of its application in practice.
\end{abstract}

Key words: bidirectional reflection; rendering; homomorphic factorization; graphic devices. 
Анотація. Функція розподілу двонаправленого відображення описує відображення світла від поверхні. Важливим є використання довільних двонаправлених відображень під час рендерингу в режимі реального часу. Метою роботи є підвищення реалістичності формування гладких поверхонь 3 використанням факторизації функції розподілу двонаправленого відображення в тривимірних сценах, що дає змогу адаптувати процес для використання сучасними графічними апаратними процесорами. Метод стиснення уявлення двонаправленого відображення має застосовуватися з використанням доступного зіставлення текстур і обчислювальних можливостей графічних процесорів. Наукова новизна роботи полягає в запропонованому чисельному методі гомоморфної факторизації, за допомогою якого можна розкласти довільні двонаправлені відображення на частини двох або більше факторів менших розмірностей, кожна 3 яких залежить від інтерпольованих геометричних параметрів. Порівняно 3 відомим методом факторизації на основі розкладання сингулярних значень запропонований метод генерує факторизацію тільки з позитивними факторами. Ця відмінність має велике практичне значення, оскільки робить його більш відповідним для сучасних графічних апаратних процесорів, забезпечуючи контроль над гладкістю результуючої поверхні, мінімізуючи відносну, а не абсолютну помилку, і може мати справу з розсіяними, розрідженими даними без окремого алгоритму вибірки та інтерполяції. Фізичний коефіцієнт відбиття поверхні може бути змодельований локально за допомогою двонаправленого методу функції розподілу відбивної здатності. Для однорідної поверхні вихідне випромінювання від точки в певному напрямку може бути розраховане з використанням інтеграла вхідного випромінювання над усіма вхідними напрямками. Чисельний підхід, заснований на сингулярному розкладанні, будує апроксимацію ряду, що складається з суми кількох добутків функцій. На відміну від чисельного підходу, в запропонованому методі використовується чистий добуток функцій у разі факторизації. Проєкції функцій визначають параметризацію множників відносно оригінальної параметризації двонаправленого відображення. Проєкції функцій можуть бути також нелінійними. Запропоноване роздільне уявлення функції уникає негативних чисел, що дає змогу використовувати прості і зручні в обчисленнях параметризації. Це забезпечує гнучкість запропонованого методу і зумовлює легкість його застосування на практиці.

Ключові слова: двонаправлене відображення; рендеринг; гомоморфна факторизація; графічні апарати.

Аннотация. Функция распределения двунаправленного отражения описывает отражение света от поверхности. Важным является использование произвольных двунаправленных отражений при рендеринге в режиме реального времени. Целью работы является повышение реалистичности формирования гладких поверхностей с использованием факторизации функции распределения двунаправленного отражения в трёхмерных сценах, что позволяет адаптировать процесс для использования современными графическими аппаратными процессорами. Метод сжатия представления двунаправленного отражения должен применяться с использованием доступного сопоставления текстур и вычислительных возможностей графических процессоров. Научная новизна работы заключается в разработке метода гомоморфной факторизации, с помощью которого можно разложить произвольные двунаправленные отражения на произведения двух или более факторов меньших размерностей, каждый из которых зависит от интерполированных геометрических параметров. По сравнению с известным методом факторизации на основе разложения сингулярных значений предлагаемый метод генерирует факторизацию только с положительными факторами. Это отличие имеет важное практическое значение, поскольку делает его более подходящим для современных графических аппаратных процессоров, обеспечивая контроль над гладкостью результирующей поверхности, минимизируя относительную, а не абсолютную ошибку, и может иметь дело с рассеянными, разреженными данными без отдельного алгоритма выборки и интерполяции. Физический коэффициент отражения поверхности может быть смоделирован локально с помощью двунаправленного метода функции распределения отражательной способности. Для однородной поверхности исходящее излучение от точки в определённом направлении может быть вычислено с использованием интеграла входящего излучения над всеми входящими направлениями. Численный подход, основанный на сингулярном разложении, строит аппроксимацию ряда, состоящую из суммы нескольких произведений функций. В отличие от численного подхода, в предлагаемом методе используется чистое произведение функций при факторизации. Проекции функций определяют параметризацию множителей по отношению к оригинальной параметризации двунаправленного отражения. Проекции функций могут быть также нелинейными. Предложенное разделяемое представление функции избегает отрицательных чисел, что разрешает использовать простые и легко вычисляемые параметризации. Это обеспечивает гибкость предложенного метода и обусловливает лёгкость его применения на практике.

Ключевые слова: двунаправленное отражение; рендеринг; гомоморфная факторизация; графические аппараты. 


\section{КОМП'ЮТЕРНІ НАУКИ ТА ІНФОРМАЦІЙНІ ТЕХНОЛОГІЇ №1ロ 2020}

\section{ПОСТАНОВКА ЗАДАЧИ}

Модель освещения Фонга в настоящее время реализована в аппаратных геометрических процессорах, однако при рендеринге имеют место незначительные различия в отражении для различных материалов. Тем не менее вычислительные возможности существующих графических конвейеров могут быть использованы для оценки альтернативных моделей освещения. В данной работе представлен метод, использующий текстуры для попиксельной оценки физически обоснованных моделей освещения. Метод позволяет отображать модели отражения широкого диапазона для различных материалов, используя только несколько текстур поиска и умножения.

\section{АНАЛИЗ ПОСЛЕДНИХ ИССЛЕДОВАНИЙ И ПУБЛИКАЦИЙ}

Практические представления двунаправленного отражения для рендеринга в реальном времени в настоящее время подразделяются на три основные категории: базисное суммирование, отображение среды и факторизация. Существует, однако, значительное количество методов, в которых эти категории частично совпадают, и зачастую их можно использовать синергетически.

Подходы суммирования базиса, представляющего двунаправленное отражение, используют сумму более простых функций. Например, двунаправленное отражение может быть представлено с помощью суммы [1] или обобщённых долей Фонга [2; 3]. В общем может потребоваться большое количество долей для аппроксимации данной функции с достаточной точностью. Если базисная функция может быть реализована эффективно, тогда эта техника может быть практичной. Например, доли Фонга, предусмотренные в модели освещения для существующих базисных функций, могут использоваться в графических конвейерах. Так как в современных аппаратных ускорителях встроенная модель освещения обычно не реализуется на уровне пикселей, подходы, основанные на факторизации и картировании окружающей среды (которые оба основаны на текстурах, поэтому оцениваются на пиксель), обычно более соответствуют для многих применений. Факторизация - это разложение функции на элементарные операции - множители. Методы представления базисных данных на основе ортонормированных разложений [4] трудно реализовать на многих современных аппаратных ускорителях [5] из-за отсутствия всесторонней поддержки используемой арифметики. Такая арифметика может быть смоделирована для стандартной графической системы при необходимости, но общее решение является дорогостоящим.

Подходы, основанные на картировании окружающей среды, могут создавать превосходное визуальное качество, но имеют проблемы с аппроксимацией, которая ограничивает их обобщение. В частности, подходы, основанные на картах окружающей среды, плохо представляют эффекты анизотропных функций. Подходы базисной функции и фильтрации карт окружающей среды могут быть объединены. Например, двунаправленное отражение можно аппроксимировать суммой базисов функции, каждый из которых радиально симметричен и поэтому подходит для фильтрации карты окружения. Подходы на основе карт окружающей среды могут сочетаться с подходами факторизации, например, для зеркальной части функции двунаправленного отражения.

Методы факторизации могут иметь дело только с точечными или направленными источниками освещения. С помощью их можно легко отрегулировать анизотропные модели отражения. Они представляют собой двунаправленные отражения с использованием функций нижних измерений (факторов), которые перемножаются [6]. Для рендеринга факторов в реальном времени, как правило, они хранятся в текстурных картах, и умножение выполняется с использованием арифметики композиции или мультитекстурирования. Вычисление факторизации можно сделать аналитически (для специфического двунаправленного отражения) или численно. Факторизация может сочетаться с окружающей средой отображения, например, путём взвешивания и возможно также предварительно отфильтрованного отображения окружающей среды с затенением, маскированием и/или коэффициентами Френеля.

\section{ОБОСОБЛЕНИЕ НЕ РЕШЕННЫХ РАНЕЕ ЧАСТЕЙ ОБЩЕЙ ПРОБЛЕМЫ}

Численный подход, основанный на сингулярном разложении, строит аппроксимацию ряда, состоящую из нескольких факторов. Даже для сложных функций, учитывающих достаточно условий, численный подход довольно точный. Однако для многих функций подход численной факторизации эффективен даже при небольшом количестве условий. Если параметризация функции выбирается тщательно, даже одно условие с двумя факторами визуально адекватно во многих случаях. Во-первых, как и другие расширения относительно ортонормированной базы, расширение требует знаковой арифметики, чтобы использоваться в полном объёме. Во-вторых, чтобы получить хорошую делимость требуется довольно сложная параметризация. В-третьих, перед применением численного метода функция должна быть реконструирована в четырёх измерениях и пересчитана на плотной регулярной сетке, которая часто бывает сложной и неудобной. В конечном счёте, приближение численного подхода минимизирует среднеквадратичную ошибку.

\section{ЦЕЛЬ ИССЛЕДОВАНИЯ}

Цель исследования заключается в повышении реалистичности формирования гладких поверхностей с использованием факторизации функции распределения двунаправленного отражения в трёхмерных сценах, что позволяет адаптировать процесс для 
использования современными графическими аппаратными процессорами.

\section{МЕТОДЫ, ОБЪЕКТ И ПРЕДМЕТ ИССЛЕДОВАНИЯ}

В работе используются методы факторизации функции распределения отражения при формировании гладких поверхностей в трёхмерных сценах. Метод сжатия представления двунаправленного отражения применяется с использованием сопоставления текстур и вычислительных возможностей графических процессоров.

Объектом исследования является процесс факторизации функции распределения двунаправленного отражения в трёхмерных сценах. Предмет исследования - методы факторизации функции распределения отражения при формировании гладких поверхностей трёхмерного изображения.

\section{ОСНОВНОЙ МАТЕРИАЛ}

Метод, представленный в данной работе, является улучшенной численной факторизацией метода для двунаправленного отражения. Его можно использовать с произвольной функцией, включая анизотропную, но ограничивается точкой или направленными источниками освещения.

Предлагаемый метод разделяемого представления функции устраняет недостатки численного подхода, так как он избегает отрицательных чисел, и в результате можно использовать гораздо более простые и более легко вычисляемые параметризации. Это делает метод более гибким и легко применяемым на практике.

Разработка метода факторизации. Физическое поверхностное отражение [6] можно моделировать локально с помощью двунаправленной функции распределения отражения. Пусть $\hat{n}$ - единичная нормаль в точке $\vec{x}$ на поверхности. Для однородной поверхности исходящую светимость $L_{0}$ из точки $\vec{x}$ в направлении $\hat{d}_{0}$ можно вычислить, использовав интеграл входящего излучения $L_{i}$ над всеми входящими направлениями $\hat{d}_{i}$. Входящее излучение должно быть утяжелено положительной проекционной площадью поверхности $\left[\hat{n} \cdot \hat{d}_{i}\right]=\max \left(0, \hat{n} \cdot \hat{d}_{i}\right)$, тогда двунаправленная функция распределения отражения $\mathrm{f}$, и измерение угла $\varsigma$ будут:

$$
L_{0}\left(\hat{d}_{0}, \vec{x}\right)=\int_{\Psi} f\left(\hat{d}_{0}, \hat{d}_{i}\right) L_{i}\left(\hat{d}_{i}, \vec{x}\right)\left[\hat{d}_{i} \cdot \hat{n}\right] d \varsigma\left(\hat{d}_{i}\right)
$$

В то время как излучения $L_{0}$ и $L_{i}$ параметризуются глобально, функция должны быть параметризована по отношению к локальной ортонормированной поверхности. Такая локальная поверхность может быть сгенерирована из нормали $\hat{n}$ и ортонормированной опорной касательной $\hat{t}$. Последний элемент локальной поверхности - нормализованный вторичный тангенс может быть сгенерирован $\widehat{s}=\hat{n} \times \widehat{t}$.
Освещение от $\mathrm{N}$ точечных источников, входящих в излучение, может быть смоделировано суммой импульсов, тогда интеграл (1) будет выглядеть следующим образом:

$$
L_{0}\left(\hat{d}_{0}, \vec{x}\right)=\sum_{j=1}^{N}\left(\hat{d}_{0}, \hat{d}_{i}^{j}\right)\left[\hat{d}_{i}^{j} \cdot \hat{n}\right]^{I} / r_{j}^{2}
$$

где $r_{j}$ - расстояние до источника света, а $I_{j}-$ его интенсивность.

Назовём это моделью локального освещения общего точечного источника. Параметры для функции двунаправленного отражения $\hat{d}_{0}$ и $\hat{d}_{i}$ имеют (в общем) четыре степени свободы. Для изотропных функций можно свести их к трём. Чтобы получить физически точное локальное освещение для точечного источника, используем вышеуказанную модель освещения с функциями двунаправленного отражения реальных материалов.

Численный подход, основанный на сингулярном разложении, строит аппроксимацию ряда, состоящую из нескольких факторов, добавленных вместе:

$$
f\left(\widehat{d}_{0}, \widehat{d}_{i}\right)=\sum_{k=1}^{K} u_{k}\left(\eta_{u}\left(\hat{d}_{0}, \widehat{d}_{i}\right)\right) v_{k}\left(\eta_{v}\left(\widehat{d}_{0}, \widehat{d}_{i}\right)\right) .
$$

Логарифмическое преобразование. Вместо использования суммы в уравнении 3 предлагаемый метод аппроксимирует функцию $\mathrm{f}$ с использованием произведения произвольного числа положительных факторов:

$$
f\left(\hat{d}_{0}, \hat{d}_{i}\right) \approx \prod_{k=1}^{K} \lambda_{k}\left(\eta_{k}\left(\hat{d}_{0}, \hat{d}_{i}\right)\right)
$$

где $\lambda_{k}$ - двумерные функции (в конечном счёте, для хранения в 2D текстурных картах), и $\eta_{k}$ : - функции проекции, связанные с каждой картой. Нелинейная проекция функции определяет параметризацию факторов относительно исходной параметризации функции двунаправленного отражения.

Логарифмируя обе части уравнения 4, приходим к следующей линейной задаче приближения данных:

$$
\log f\left(\hat{d}_{0}, \hat{d}_{i}\right) \approx \sum_{k=1}^{K} \log \lambda_{k}\left(\eta_{k}\left(\hat{d}_{0}, \hat{d}_{i}\right)\right) .
$$

Как только решим это преобразование, сможем преобразовать результат решения исходной нелинейной задачи путём возведения в степень. Логарифмический/экспоненциальный гомоморфизм упрощает проблему, а также гарантирует, что результат будет положительным, если решим уравнение 5 .

Предлагаемый численный метод может определить оптимальные значения для произвольного ряда факторов и может получить сколь угодно точные приближения с соответственно независимыми функциями проекции. Для достижения наилучших результатов важно выбрать проекцию функции k, совместимую с функциями и направлениями наибольших изменений функции $\mathrm{f}$. 


\section{КОМП'ЮТЕРНІ НАУКИ ТА ІНФОРМАЦІЙНІ ТЕХНОЛОГІЇ №1ロ 2020}

Математику и численные методы представим аналогично алгоритмам, используемым в компьютерной аксиальной томографии, таким как фильтрованная обратная проекция. Фильтрованное обратное проецирование может реконструировать многомерную функцию с произвольной точностью, учитывая достаточно независимые проекции нижних измерений. Однако вместо того, чтобы восстанавливать функцию из проекций, находим набор проекций для наилучшего восстановления заданной функции. Также делаем однородный и плотный набор проекционных функций.

Параметризация. Несмотря на потенциальную общность предлагаемого метода, в данной работе ограничимся (в основном) тремя факторами приблизительных функций представления и очень простой параметризацией, которая использует одну или две текстурные карты:

$$
\begin{aligned}
\quad f\left(\hat{d}_{0}, \hat{d}_{i}\right) & \approx \lambda\left(\hat{d}_{0}\right) \pi(\hat{m}) \lambda\left(\hat{d}_{i}\right) \\
\text { где } & \pi(\hat{m})=\operatorname{norm}\left(\hat{d}_{0}+\hat{d}_{i}\right) .
\end{aligned}
$$

Эта параметризация основана на предположениях, что затенение, маскирование и распределение - вариации коэффициента отражения, а также что затенение и маскирование независимы. Конечно, есть и другие влияния, которые вносят вклад в отражение, - это рассеяние, интерференция, эффект Френеля. Однако предлагаемая численная методика будет использовать доступные степени свободы, чтобы соответствовать физике явлений, насколько это возможно. Например, изменение цвета, вызванного рассеянием и интерференцией будут автоматически аппроксимироваться функциями, которые изменяются в зависимости от направления выбранной параметризации. Не исключено, что в будущем появятся и другие параметризации и факторизации, доминирующая вариация которых будет обусловлена другими механизмами. Возможно, что оптимизированные параметризации можно будет найти автоматически. Например, с помощью дополнительных факторов, векторов параметризации или нормализованных аффинных комбинаций.

Тем не менее конкретное целевое представление, приведённое в уравнении 6, имеет ряд преимуществ. Приближение всегда будет удовлетворять реципрокальности Гельмгольца. Представление требует только две текстурные карты, что минимизирует затраты на хранение и разрешение многих функций для использования в сценах даже в недорогих системах. Хотя три фактора необходимы в итоге, только два фактора зависят от направления источника света, что упрощает реализацию в системах, допускающих только два поиска текстуры за один проход, когда требуется несколько источников света. Параметризация также легко вычисляется для поверхностей с преобладанием затенения и маскирования, а также для тех, в которых доминируют распределения, это охватывает большие классы материалов.

\section{ОБСУЖДЕНИЕ ПОЛУЧЕННЫХ РЕЗУЛЬТАТОВ}

Графические процессоры, в основном специализированные на SIMD/векторных компьютерах, способны с ограниченной точностью к численным вычислениям. В данной задаче используем стандартное OpenGL с мультитекстурированием в качестве вычислительной модели. В реализации используется также в Nvidia расширение вершинного шейдера. Для хранения факторов представления карты текстуры параметризованы по направлению полушарий, это позволяет делать хорошую интерполяцию. Также выполняется разложение на множители с помощью параболического отображения соответствующих единичных векторов. Параболические карты работают со всеми аппаратными ускорителями, правильно реализующими проективные преобразования и интерполяцию координат текстуры. Результирующие 2D-изображения могут быть повторно объединены в карты кубов, если оборудование поддерживает их.

Приближение визуально сравнивается с использованием функции двунаправленного отражения на рисунке 1. Для анализа ошибок использована аналитическая модель анизотропного отражения, которая не учитывает многократное рассеяние, но которая учитывает различные эффективные нормальные распределения из-за затенения и маскирования.

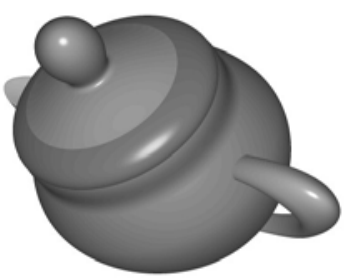

a)

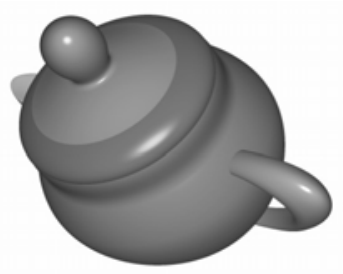

б)
Рис. 1: а) использование фактических значений функции двунаправленного отражения в вершинах тесселированной модели, выполненной с затенением Фонга; б) приближение с использованием факторизации

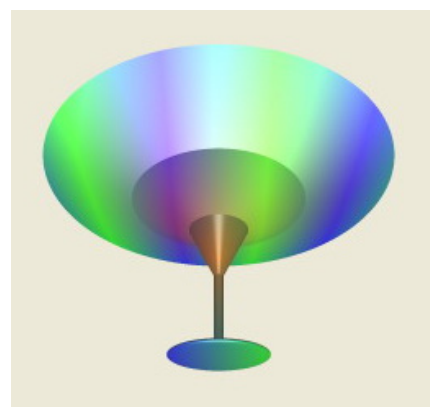

Рис. 2. Приближение с использованием факторизации 


\section{ЗАКЛЮЧЕНИЕ}

Представлен метод вычисления функции двунаправленного отражения в реальном времени для освещения локальных анизотропных материалов из точечного источника света с использованием графических процессоров. Представление функции может храниться в двухмерных (со средним разрешением), картах текстуры и одной карте куба. Поэтому сцена может со- держать модели со многими различными функциями. Метод факторизации имеет несколько преимуществ перед разложением сингулярных значений, включая фазу факторизации, автоматического заполнения, совместимость с разреженными, рассеянными данными функции, относительной минимизации, а не абсолютной ошибки, поддержку произвольных параметризаций и использование положительных факторов.

\section{REFERENCES}

[1] Luongo, A., Falster, V., Doest, M. B., Li, D., Regi, F., Zhang, Y., Tosello, G., Nielsen, J. B., Aanæs, H., Frisvad, J. R. (2017). Modeling the Anisotropic Reflectance of a Surface with Microstructure Engineered to Obtain Visible Contrast after Rotation. Reflectance of a surface with microstructure engineered to obtain visible contrast after rotation. In Proceedings of IEEE International Conference on Computer Vision Workshop (ICCVW 2017), 159-165.

[2] Romanyuk, O. N., Chornui, A. V. (2006). High-performance methods and tools for painting 3D objects. Monograph. Vinnytsia: UNIVERSUM-Vinnytsia. 190 p. [in Ukrainian].

[3] Romanyuk, O. N. (2008). Classification of distributive functions of surface reflectance. Scientific works of Donetsk National Technical University. Ser.: Computer science, cybernetics and computer engineering. Pub. 9. pp. 145-151 [in Ukrainian].

[4] Stollnitz, E., DeRose, T., Salesin, D. (1996). Wavelets for Computer Graphics: Theory and Applications. Morgan Kaufmann Publishers.

[5] Romanyuk, O. N., Dovgaliuk, R. U., Olijnuk, S. V. (2011). Classification of graphic video adapters. Scientific works of Donetsk National Technical University. Ser.: Computer science, cybernetics and computer engineering. Pub. 14. Pp. 211-215 [in Ukrainian].

[6] Cohen, M.F., Wallace, J.R. (1993). Radiosity and Realistic Image Synthesis, Academic Press.

\section{СПИСОК ИСПОЛЬЗОВАННОЙ ЛИТЕРАТУРЫ}

[1] Luongo, A., Falster, V., Doest, M. B., Li, D., Regi, F., Zhang, Y., Tosello, G., Nielsen, J. B., Aanæs, H., Frisvad, J. R. (2017). Modeling the Anisotropic Reflectance of a Surface with Microstructure Engineered to Obtain Visible Contrast after Rotation. Reflectance of a surface with microstructure engineered to obtain visible contrast after rotation. In Proceedings of IEEE International Conference on Computer Vision Workshop (ICCVW 2017), pp. 159-165.

[2] Романюк, О. Н., Чорний, А. В. (2006). Високопродуктивні методи та засоби зафарбовування тривимірних графічних об’єктів : монографія. Вінниця : УНІВЕСУМ-Вінниця. 190 с.

[3] Романюк, О.Н.(2008). Класифікація дистрибутивних функцій відбивної здатності поверхні. Наукові праці Донецького начіонального технічного університету. Сер.: Інформатика, кібернетика та обчислювальна техніка. Вип. 9. C. $145-151$.

[4] Stollnitz, E., DeRose, T., Salesin, D. (1996). Wavelets for Computer Graphics: Theory and Applications. Morgan Kaufmann Publishers.

[5] Романюк, О. Н., Довгалюк, Р. Ю., Олійник, С. В. (2011). Класифікація графічних відеоадаптерів. Наукові праиі Донецького начіонального технічного університету. Сер.: Інформатика, кібернетика та обчислювальна техніка. Вип. 14. С. 211-215.

[6] Cohen, M. F., Wallace, J. R. (1993). Radiosity and Realistic Image Synthesis, Academic Press. 